\title{
Comportamientos Tecnológicos en el Sector Olivícola de la Provincia de La Rioja. El rol del Sistema Local de Innovación.
}

\section{Technological behaviours in the Olive Sector in La Rioja province. The role of the Local Innovation System.}

ARTÍCULO

\author{
Gabriela Starobinsky \\ Universidad Nacional de Chilecito, Departamento de Ciencias Sociales, Jurídicas y \\ Económicas, Argentina. \\ Contacto: gstarobinsky@gmail.com / gstarobinsky@undec.edu.ar
}

Recibido: marzo de 2018

Aceptado: mayo de 2018

\section{Resumen}

La presente investigación aborda el rol del Sistema Local de Innovación (SLI) sobre los esfuerzos innovativos de las firmas para el sector olivícola de La Rioja, Argentina. El análisis empírico se efectúa en base a una encuesta conducida por la Universidad Nacional de Chilecito en 2012, para cuyo procesamiento se construyen regresiones QuasiPoisson y Logit Binomial. Los resultados muestran que mayor cantidad de relaciones de cooperación promueven mayores esfuerzos tecnológicos. Sin embargo, la heterogeneidad entre las firmas y las debilidades del SLI limitan su desempeño.

Palabras clave: Esfuerzos Innovativos; Interacciones; Sistema Local de Innovación; Sector Olivícola (Clasificación JEL: D21, O18, O30, R19)

\begin{abstract}
The present research studies the role of the Local Innovation System (LIS) in relation to firms' innovation efforts for the olive sector in La Rioja, Argentina. Empirical analysis is based on information gained from a survey conducted by the National University of Chilecito in 2012. Quasi-Poisson and Binomial Logistic regressions are built in order to deal with statistical data. The main findings states that the more cooperative relations firms establish, the more technological efforts they make. However, heterogeneity amongst firms and LIS' weaknesses limit their performance.
\end{abstract}

Keywords: Innovation Efforts; Interactions; Local Innovation System; Olive Sector. 


\section{Introducción}

La consolidación en las últimas décadas de un nuevo paradigma tecno-económico a nivel mundial, intensivo en la aplicación de conocimientos a la producción, conlleva a que los niveles de productividad y competitividad alcanzados por las firmas, las regiones y los países, se encuentren fuertemente relacionados con las capacidades tecnológicas y los esfuerzos requeridos para generar innovaciones. Sin embargo, las regiones de menor desarrollo relativo enfrentan obstáculos particulares a la hora de encarar dichos comportamientos (David y Foray, 2002).

En ese sentido, el propósito general del presente trabajo es contribuir a la comprensión de los procesos locales de innovación en regiones en desarrollo, en las que coexisten actores heterogéneos y preponderan firmas pequeñas y medianas. Particularmente, se estudia en profundidad el caso del sector olivícola de la provincia de La Rioja, Argentina.

Para ello, se aborda la problemática desde el enfoque conceptual del Sistema Local de Innovación (SLI) para el análisis de los rasgos característicos de los procesos de desarrollo tecnológico en regiones particulares. Se pretende evaluar así la influencia del entorno local sobre el comportamiento tecnológico de las firmas del sector olivícola.

El análisis empírico se desarrolla sobre la base de información estadística recopilada por medio de una encuesta específica conducida por la Universidad Nacional de Chilecito en el año 2012. Luego, para el abordaje metodológico se construyen Modelos Econométricos de Respuesta Cualitativa para analizar la relación entre las características de las firmas, sus relaciones con el entorno y las actividades innovativas que éstas realizan. Así, se construyen una serie de modelos tomando las regresiones Quasi-Poisson y Logit Binomial.

El trabajo se encuentra estructurado de la siguiente manera. En primer lugar, se plantean el marco conceptual y los antecedentes del problema de investigación. En segundo lugar, se presentan el caso de estudio junto con la metodología para su análisis. Por último, se postulan los resultados y conclusiones del mismo.

\section{Marco Conceptual y Antecedentes de Investigación}

\section{a) EI Rol de los Sistemas Locales de Innovación}

El marco conceptual general en el cual se encuadra la presente investigación se basa en la corriente de pensamiento del evolucionismo económico, para la cual el avance tecnológico cumple un rol fundamental en la competitividad tanto de las firmas como de las regiones, y por ende en el desarrollo socio-económico. En particular, sostiene una concepción sistémica y acumulativa de la innovación, que requiere de esfuerzos continuos 
y colectivos a nivel local para construir capacidades tecnológicas endógenas (Lundvall, 1997).

El enfoque sustantivo para abordar la problemática de la relación entre las características del entorno local y el comportamiento tecnológico de las empresas, se basa en la concepción del Sistema Local de Innovación (SLI). Principalmente, se destaca el carácter interactivo de la innovación, la generación de resultados y para ello, la incorporación de nuevos conocimientos no son triviales sino que las firmas deben contar con una serie de capacidades (de absorción, adaptación, diseño, identificación de tecnologías, y vinculación) para encarar los esfuerzos necesarios. Dichas habilidades no son intrínsecas a la empresa, sino que se construyen a lo largo de aprendizajes y asimilación de saberes tácitos y codificados (López, 2002).

Así, la conceptualización del SLI sostiene que los procesos de cambio tecnológico e innovación se encuentran social, cultural y territorialmente enraizados, y lejos de ser lineales en cada sistema presentan características específicas (Lundvall, 1997). Cada entorno local adopta especificidades únicas, dadas por su cultura, sus instituciones y organizaciones, su estructura económica, social y política, que operan en algún sentido sobre el desempeño innovador (Tödtling, Lehner y Kaufmann, 2008). Entre los principales fenómenos que se generan se encuentra la sinergia y eficiencia colectiva, las economías de aglomeración y asociación, y el aprendizaje por interacción (Cassiolato, Lastres y Maciel, 2003).

De este modo, la conformación de sistemas locales en donde se desarrollan redes de cooperación, aprendizajes por interacción, difusión y transferencia tecnológica, promueven procesos colectivos que facilitan la construcción de capacidades, la innovación y la reducción de incertidumbre. Por lo tanto, es en los entornos locales donde se concentran las vinculaciones entre agentes co-localizados que fomentan esfuerzos y procesos innovativos. El desarrollo de actividades innovativas por parte de las firmas depende no sólo de sus propios esfuerzos sino de su capacidad para relacionarse e interactuar con otras empresas y organismos de ciencia y tecnología (СуT) (Cassiolato et al., 2003). Puntualmente, las vinculaciones facilitan el flujo de conocimientos, refuerzan la confianza entre los actores, incrementan los derrames locales, favorecen las economías externas, la transferencia de tecnologías, reducen los costos de transacción, y disminuyen la incertidumbre (Tödtling et al., 2008).

Consecuentemente, las condiciones del entorno permiten en mayor o menor medida desencadenar y fortalecer la difusión y transferencia de saberes codificados y tácitos, incrementar las externalidades, y fomentar procesos de aprendizajes tecnológicos que devienen en el desarrollo y acumulación de capacidades. Las características del SLI tales como la cantidad y tipo de actores, la presencia de traductores, la circulación y el nivel de apropiación del conocimiento, el grado de vinculación entre los actores, las políticas de 
promoción de ciencia, tecnología e innovación (CTI), y la existencia de redes de cooperación, influyen sobre las posibilidades para construir competencias y encarar procesos innovativos (Yoguel, Borelloy Erbes, 2009).

Sin embargo, no en todos los sistemas es factible para cualquier tipo de empresa poder aprovechar las externalidades locales. En ese sentido, Yoguel y Boscherini (2001) plantean que en contextos donde se desencadenan procesos de eficiencia y aprendizaje colectivos el tamaño de las firmas no resulta determinante en la construcción de capacidades innovativas, ya que los factores positivos del sistema resultan beneficiosos para superar sus propias limitaciones. Por el contrario, en aquellos SLI en los cuales se generan menores sinergias, el umbral mínimo de destrezas requerido es mayor, y las pequeñas empresas no logran aprovechar las oportunidades disponibles.

En ese sentido, respecto a los sistemas de regiones de menor desarrollo relativo se destacan debilidades tales como, niveles de competencias tecnológicas bajas por parte de empresas y organismos; recursos humanos con escasas calificaciones; falta de recursos financieros; limitaciones para acceder a políticas públicas; y pocas interacciones e intercambios de conocimientos, las cuales condicionan la virtuosidad de los sistemas locales (Yoguel et al., 2009).

\section{b) Casos de Entornos Virtuosos, Interacciones e Innovación}

A lo largo de las últimas dos décadas, el reconocimiento del carácter sistémico y territorialmente enraizado del proceso de innovación da lugar a una serie de investigaciones empíricas que aportan al conocimiento de las particularidades que adopta en diversas regiones del mundo. Todas ellas analizan la influencia del entorno local, la proximidad geográfica y la articulación entre actores, sobre las capacidades tecnológicas de las firmas, las innovaciones y el desarrollo económico.

Por un lado, para Europa se destacan los estudios de los distritos industriales de Pequeñas y Medianas Empresas (Pymes) en Italia, como Camagni y Capello (1997), quienes sobre la base de un análisis estadístico sostienen que los factores de la atmósfera local tales como la cercanía física y cultural, el contacto interpersonal, el intercambio de información y conocimientos, la movilidad de recursos, la reducción de incertidumbre, y la conformación de redes sinérgicas, resultan relevantes para potenciar los procesos innovativos locales.

En el mismo sentido, los trabajos para otras regiones de Europa como el de Natário, Braga, Couto y Tiago (2012) en una investigación para Portugal del año 2006 que plantea la comparación de la dinámica innovativa de distintos clusters, concluye que los resultados de cada región se encuentran asociados tanto a los esfuerzos internos de las firmas (Gasto en I+D) como a las redes de relaciones que entretejen. 
Asimismo, se puede mencionar las conclusiones de Asheim y Coenen (2005) para los clusters nórdicos de fabricación de muebles, comunicaciones inalámbricas, electrónica y alimentos. En base a un análisis descriptivo pormenorizado los autores sostienen que en todos los casos los procesos de innovación se encuentran relacionados con las características del entorno local dadas por el flujo de conocimiento tácito, la interacción directa entre actores, la cooperación con clientes y proveedores, la cultura regional (valores, normas compartidas y el nivel de confianza), la presencia de recursos humanos calificados, y el acceso al conocimiento científico.

Por otra parte, existen diversos trabajos enfocados en el análisis de las vinculaciones entre actores y la conformación de sistemas de innovación en economías en desarrollo tales como Intarakumnerd y Vang (2006) y Hassink (2002) en los países del sudeste asiático. Éstos señalan cómo la absorción de tecnologías y adaptaciones creativas se generan sobre la base de un conjunto de capacidades a nivel micro y mesoeconómico que se construyen de manera evolutiva y colectiva.

Luego, se destaca un conjunto de antecedentes que estudian la problemática de la vinculación entre firmas y actores del SLI, y su relación con el desempeño innovador, por medio de Modelos de Respuesta Cualitativa. Por ejemplo, Fritsch y Franke (2004) comparan tres regiones de Alemania (Baden, Hannover y Sajonia) y encuentran que la ubicación geográfica influye sobre los esfuerzos innovativos de las firmas, siendo que aquellas de las regiones occidentales presentan mayores probabilidades de registrar al menos una patente y de hacerlo con mayor intensidad. Respecto a los derrames tecnológicos medidos por el gasto en I+D de otros actores, y por la interacción entre empresas y organizaciones, se observa que impactan positivamente sobre los resultados de las firmas. Tomando en cuenta la misma muestra Fritsch (2001) muestra que la cantidad de nexos establecidos con otros organismos se relaciona con el desempeño innovador.

Adicionalmente, Fritsch y Slavtchev (2011) abordan para regiones de Alemania el impacto de las características del SLI, y la colaboración entre actores sobre el número de patentes obtenidas. Los principales resultados muestran el efecto positivo y significativo de los derrames de conocimiento, la presencia de universidades y organismos de investigación, la congruencia de los campos de estudio entre sector público y privado, y la intensidad de las interacciones.

En el mismo sentido, Kaufman y Tödtling (2000) conducen una investigación semejante para las regiones de Europa, Gales (Reino Unido), Región Valona (Bélgica), Baden (Alemania), Estiria (Austria), País Vasco (España), Aveiro (Portugal), y Tampere (Finlandia). Los resultados exhiben que los principales obstáculos que limitan las interconexiones entre actores son la falta de instituciones científicas, la incongruencia entre las necesidades de las empresas y la oferta tecnológica, la presencia de barreras en 
la comunicación, la existencia de normas e intereses disímiles, y la reticencia a cooperar por parte de las firmas.

\section{c) Comportamientos Tecnológicos y Sistemas Locales en América Latina y Argentina}

A nivel regional, si bien para América Latina el estudio de los Sistemas Locales de Innovación es un terreno fértil y poco explorado, se encuentran investigaciones que analizan en profundidad las características de los procesos de innovación y su relación con las particularidades del entorno para regiones de diversos países. Se destaca así el trabajo de Jiménez, Fernández de Lucio y Menéndez (2011) que presenta estudios en profundidad para Brasil, México, Colombia y Chile.

Para el caso de Brasil toman en cuenta los estados de Santa Catarina y Ceará, principalmente se destaca una gran debilidad en la articulación entre el sector industrial y los organismos de СуТ. La infraestructura de СуТ no apunta a resolver las problemáticas tecnológicas de las empresas locales, por lo que exceptuando un conjunto de firmas grandes que cuenta con capacidades innovativas elevadas, la mayoría enfrentan dificultades para encarar avances tecnológicos (Jiménez et al. 2011).

Para el estudio de los SLI chilenos se exploran las regiones de Araucanía, Los Ríos y Los Lagos. En Araucanía se encuentran difundidas las redes de innovación, las agencias de interfaz y programas de cooperación tecnológica, siendo los sectores de turismo y acuícola los de mayor dinamismo. Sin embargo, se observan algunas limitaciones como la falta de inclusión de las microempresas y la débil participación de las universidades. Luego, en Los Lagos la Universidad Austral de Chile adopta un rol activo en la vinculación con empresas acuícolas por medio de proyectos de investigación conjuntos. Por el contrario, en Los Ríos las articulaciones entre actores son muy bajas y enfrentan, a su vez, serias limitaciones en términos de recursos. Para el caso de Chile los autores destacan como uno de los principales obstáculos para innovar la horizontalidad de los instrumentos de CTI, que se orientan a la resolución de problemáticas y promoción de acciones a nivel nacional (Jiménez et al., 2011).

De manera adicional, el estudio ALIAS (2011) presenta un análisis para la región Biobío en Chile donde existe una estructura empresarial heterogénea. Entre las principales debilidades del SLI se identifican la escaza cooperación entre organizaciones de СуТ y PyMes, la divergencia entre la demanda tecnológica y la oferta, la débil coordinación de las acciones de transferencia y, la dificultad para acceder a instrumentos de promoción por parte de pequeñas empresas.

Por otra parte, en Colombia en los departamentos del Valle del Cauca y de Antioquia se desempeñan una serie de organizaciones nacionales y regionales de interfaz que promueven la innovación y las redes cooperativas. Así, hacia su interior se generan fuertes 
conexiones que facilitan los procesos de aprendizaje colectivos, la difusión de conocimientos $\mathrm{y}$, el incremento de la competitividad. Cabe señalar que en Colombia la planificación de políticas de CTI se orienta a nivel regional, atendiendo a la heterogeneidad presente entre éstas (Jiménez et al., 2011).

Otro estudio sobre la dinámica local de innovación a mencionar es el de Piñero, Arzola y Rodríguez (2012) para la región de Bolívar en Venezuela. Los autores detectan que las condiciones del entorno y las políticas de promoción no tienen un impacto positivo, dado el escaso financiamiento para promover actividades innovativas y, la dificultad por parte de pequeñas empresas para acceder a los programas existentes. Adicionalmente, hacia el interior del SLI se identifican exiguas vinculaciones entre empresas, centros de I+D y universidades.

Para el caso de Argentina, uno de los principales casos de estudio es el de la localidad de Rafaela en la provincia de Santa Fe, en donde se concentra un conjunto de empresas competitivas tanto a nivel local como internacional, inmersas en un entramado institucional denso y articulado. Boscherini, López y Yoguel (1998) sostienen que los resultados alcanzados por las empresas no sólo se deben a sus esfuerzos internos, sino también a la dinámica del ambiente. La presencia de instituciones públicas y privadas, el accionar del gobierno, la existencia de recursos humanos calificados, la idiosincrasia empresaria, la generación de externalidades y la articulación entre actores, construyen una atmósfera industrial virtuosa. Respecto a las vinculaciones resaltan que la cantidad de conexiones es un factor influyente sobre las capacidades de las firmas.

Adicionalmente, Gennero de Rearte, Lanariy Alegre (2006) realizan un análisis similar para Mar del Plata. En este caso, las firmas que establecen articulaciones con actores del entorno local consideran de suma importancia dichos nexos y el impacto de éstos sobre sus capacidades tecnológicas. Además, dado que la capacidad innovativa no parece estar relacionada directamente con el tamaño de la firma, puede suponerse que el ambiente local favorece a las empresas medianas permitiéndoles superar las barreras intrínsecas a su dimensión.

A su vez, un importante trabajo de Yoguel, Borello y Erbes (2006) aborda la caracterización y comparación de los Sistemas Locales de Innovación de Córdoba, Rafaela, Rosario, Tucumán, Salta y Jujuy, a través de información recopilada por encuestas a empresas. En particular, en Córdoba si bien existe un sistema científicotecnológico desarrollado, se observa que las interacciones entre actores son insuficientes, y se concentran en intercambios de información. Sin embargo, existen experiencias de cooperación exitosas que favorecen la construcción de capacidades innovativas en los sectores de TICs, equipamiento médico y automotriz.

Luego, los autores presentan en profundidad el SLI de Rosario, el cual se caracteriza por contar con una estructura educativa, científica y tecnológica densa, y un conjunto de 
programas y agencias para el desarrollo regional de carácter mixto. No obstante, en los casos de Rosario y Córdoba existe una relación entre el tamaño de las empresas, su desempeño y capacidad para aprovechar las externalidades positivas del entorno. De este modo, son las empresas grandes y medianas las que participan en redes locales y alcanzan un mayor desarrollo tecnológico.

Por último, se analizan los sistemas locales de tres provincias del noroeste argentino de menor desarrollo económico e industrial: Tucumán, Salta y Jujuy. En términos generales se encuentran esfuerzos cooperativos poco difundidos; desarticulación de los sistemas, falta de interacción entre actores, escaso aprovechamiento de instrumentos de promoción, ausencia de políticas específicas de CTI y, débiles competencias tecnológicas (Yoguel et al., 2006).

Adicionalmente, se pueden destacar una serie de trabajos que estudian el desempeño y la dinámica innovativa de grupos de PyMes para distintas provincias. Así, la Tesis Doctoral de Robert (2012) da cuenta de la heterogeneidad en el desempeño de las firmas tanto entre sectores y áreas geográficas como hacia el interior de las mismas. La investigación muestra la importancia de la complementariedad de los conocimientos y capacidades de las firmas con aquellos de carácter externo para el aprovechamiento de las externalidades del entorno que facilitan los procesos de innovación. Entre los principales resultados, la autora demuestra la existencia de círculos virtuosos entre las capacidades de absorción, las interacciones con otros actores y, el tamaño de las firmas.

De esta forma, los antecedentes presentados corroboran lo analizado en el marco conceptual, los entornos en donde se generan relaciones de cooperación y sinergias entre los actores del sistema favorecen el desempeño económico e innovativo de las firmas. Así, las interacciones para el intercambio de conocimiento y transferencia de tecnologías adquieren relevancia tanto en economías maduras, donde preponderan los procesos de generación de innovaciones radicales, como en aquellas en vías de desarrollo, en las que las firmas encaran primordialmente la construcción de capacidades de absorción y mejoras adaptativas e incrementales.

En consecuencia, los esfuerzos internos y las condiciones del entorno, tales como la cercanía geográfica, la infraestructura científico-tecnológica, los instrumentos de financiamiento $\mathrm{y}$, los derrames de conocimiento, influyen sobre las oportunidades de mejorar el desempeño tecnológico de las firmas. Sin embargo, no son procesos que se generan automáticamente, sino que también se encuentran obstáculos como la inadecuación de la oferta tecnológica, problemas de comunicación y diseño de políticas, divergencias en los intereses y tiempos de respuesta.

\section{El Sector Olivícola en la provincia de La Rioja}


La Rioja es una de las principales provincias olivícolas del país, ocupa el primer lugar en términos de hectáreas implantadas y producción, y es a su vez, la primera exportadora de aceite de oliva y la segunda de aceitunas de mesa. Asimismo, el sector representa una parte importante de la estructura productiva de la provincia, es la principal actividad agropecuaria superando ampliamente la producción y las has. cultivadas de vid y nogal. En términos de exportaciones, el complejo representa el $20 \%$ del total provincial (DNPR 2016, Day 2013).

El desarrollo de la actividad a nivel regional se relaciona directamente con la aplicación durante la década de 1990 de la Ley de Diferimientos Impositivos (Ley $N^{\circ}$ 22.021). Dados los beneficios impositivos, se observa en dichos años la radicación de nuevas explotaciones olivícolas y un importante incremento tanto de la producción primaria como secundaria en las provincias de La Rioja, Catamarca y San Juan. En particular, se trata de capitales nacionales extra-locales que establecen grandes y medianos emprendimientos en la región (Matías, Molina, Aybar, Ladux y J. Ortiz, 2012).

En consecuencia, se genera una re-estructuración del sector olivícola, que implica un aumento del tamaño promedio de la explotación, la disminución de los marcos de plantación, la adopción de nuevas tecnologías y mecanización de labores culturales y el incremento de los rindes promedios de la actividad. Así, el sector se caracteriza por la existencia de distintos tipos de unidades productivas que operan en condiciones heterogéneas en cuanto a la escala de producción, las prácticas tecnológicas y productivas, y por ende, la productividad por hectárea (Matías et al., 2012).

Existen alrededor de 3.000 explotaciones agropecuarias productoras de olivos, entre las que conviven productores medianos y grandes tecnificados con mayores rendimientos, junto a minifundios locales, pudiéndose identificar dos tipos de modelos productivos. Por un lado, explotaciones tradicionales que utilizan mano de obra familiar, poseen un bajo nivel de tecnificación, escaso consumo de fertilizantes y agroquímicos, implementan riego superficial por inundación y cosecha manual. Por otro lado, sistemas modernos que emplean mano de obra asalariada, utilizan sistemas de riego por goteo automatizados, conducen labores de poda mecánica y aplicación de agroquímicos junto al control de plagas y enfermedades. Mientras que el rendimiento promedio del modelo tradicional es de $3 \mathrm{tn} / \mathrm{ha}$., en las explotaciones modernas alcanza entre 7,5 y 12,5 th/ha. (Vita Serman y Matías, 2013).

En cuanto a los desarrollos tecnológicos involucrados en la actividad olivícola, éstos comprenden una diversidad de técnicas y conocimientos que atraviesan la misma desde la producción primaria hasta las etapas de proceso industrial y comercialización. En ese sentido, el sector cuenta con una amplia oferta de tecnologías, y oportunidades de innovación, que abarcan desde la genética a implantar hasta el envasado y la 
comercialización, pasando por tecnologías en cosecha, riego, poda, fertilización, transporte, procesamiento y almacenamiento (Sánches, 2013).

Si bien se trata de un sector agroindustrial no muy complejo tecnológicamente, la implementación de técnicas modernas se torna fundamental a la hora de alcanzar competitividad internacional, dadas las crecientes exigencias en términos de calidad, inocuidad y trazabilidad en las industrias alimenticias. Asimismo, de su adopción depende la resolución de los problemas que enfrenta el sector tales como la escasez y el mal manejo del agua; la presencia de enfermedades y plagas; la falta de tipificación de los aceites; el bajo valor agregado; la existencia de estándares de calidad diferentes a los internacionales; y la ausencia de canales de comercialización (Sánches, 2013).

De esta forma, la brecha tecnológica presente hacia el interior del sector en todas las etapas productivas, sumado a la heterogeneidad entre las firmas, implican problemas de productividad frente a los productores de los países olivareros como España e Italia. En ese sentido, se enfrentan una serie de desafíos para la modernización del sector y consecuentes incrementos en la calidad y diferenciación de productos (Sánches 2013).

\section{Abordaje Metodológico y Resultados Econométricos}

Para el estudio de las relaciones entre las características de las firmas, las particularidades del SLI, las interacciones con otros actores, y los esfuerzos de innovación, se cuenta con información detallada y recabada por una encuesta específica denominada "Demanda Tecnológica para el Sector Olivícola de la Provincia de La Rioja", conducida por la Unidad de Vinculación Tecnológica de la Universidad Nacional de Chilecito (UNdeC, 2013). A continuación se presentan las hipótesis de trabajo que guían el análisis, para luego desarrollar el análisis descriptivo de la muestra y, por último, los resultados de los modelos econométricos construidos.

\section{a) Hipótesis de Trabajo}

H1- Las vinculaciones de las firmas dentro del SLI las motivan a realizar actividades de innovación.

$\mathrm{H} 2$ - Las debilidades presentes hacia el interior del SLI representan limitaciones para las firmas respecto a su comportamiento tecnológico.

\section{b) Caracterización Sectorial}

\section{Perfil productivo y comportamientos tecnológicos}

La muestra comprende 91 productores olivícolas distribuidos en Capital (20\%), Arauco $(37 \%)$ y Chilecito (43\%), los principales departamentos dedicados a la explotación olivarera de la provincia. Entre ellos la mayoría se dedica sólo a la actividad primaria, los 
cuales representan el $66 \%$ de las observaciones, y un $34 \%$ se encuentra integrado con el sector secundario.

Si bien el conjunto de las firmas son empresas PyMes ya que ninguna supera los 100 empleados, se las puede subclasificar por tamaño teniendo en cuenta tramos de empleo. En función de la cantidad de empleados permanentes se destaca la presencia de microempresas con hasta 5 empleados, las cuales abarcan el $52 \%$ de la muestra, el $15 \%$ corresponde a pequeñas de hasta 10 empleados, $25 \%$ a firmas medianas entre 11 y 50 y sólo un $8 \%$ cuenta con más de 50 .

De manera adicional, debido a las implicancias de la Ley de Diferimientos Impositivos, se torna relevante discriminar las explotaciones en términos del origen de la inversión. En ese sentido, existe una fuerte presencia de capitales nacionales extra-locales que representan el $52 \%$ de los productores, perteneciendo los restantes a inversiones provinciales, por lo que conviven modelos productivos tradicionales junto a plantaciones modernas.

Así, la heterogeneidad de las firmas del sector condiciona y dota de particularidades su perfil innovador y su comportamiento tecnológico. En primer lugar, se puede destacar que un 62\% -56 observaciones- de los productores encuestados afirman realizar al menos una Actividad de Innovación (Al) (Gráfico $N^{\circ} 1$ ). Aunque en promedio no se supera las dos actividades por firma aquellas grandes combinan en promedio más de tres tipos distintos. A su vez, se destaca su preponderante difusión entre empresas extra-locales y de mayor tamaño.

Gráfico N¹. Proporción de Productores que realizan Actividades de Innovación por Tamaño y Origen de la Inversión

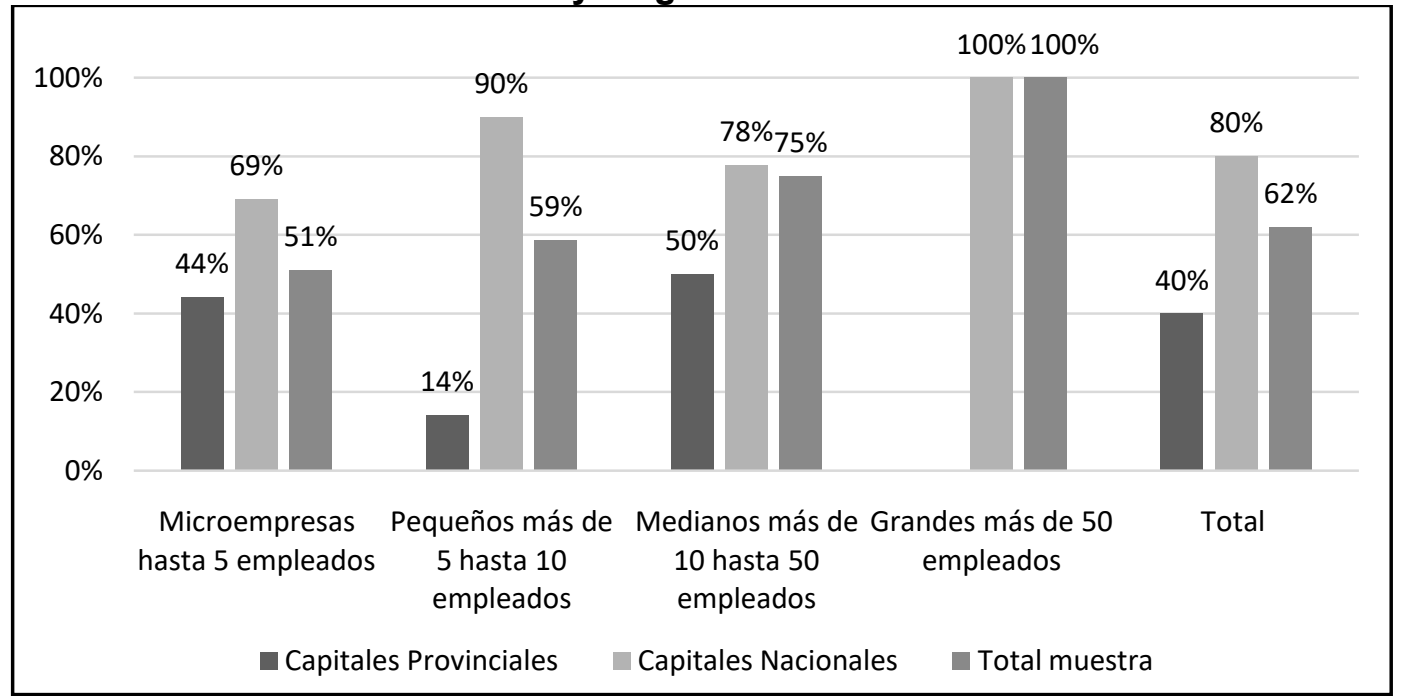

Fuente: elaboración propia en base a Encuesta "Demanda Tecnológica para el Sector Olivícola de la Provincia de La Rioja".

Luego, en cuanto a su composición, tomando la totalidad de actividades de innovación realizadas por el conjunto de productores (112), el $31 \%$ corresponde a la 
Adquisición de Maquinarias y Equipos (MyE); el 25\% a Capacitación de Recursos Humanos; el 19\% a Contratación de Maquinaria y Servicios; el 15\% a Investigación y Desarrollo; y el 10\% a incorporación de TICs y Automatización. Por otra parte, tomando en cuenta la cantidad de firmas que realiza cada tipo de Al por estrato se identifica una marcada diferencia entre aquellas micro y grandes, encontrándose todas las actividades mayormente difundidas entre estas últimas. De esta forma, la difusión y diversidad de las actividades de innovación es mayor entre las empresas medianas y grandes.

Si bien se identifican una serie de esfuerzos relacionados al desarrollo tecnológico, los resultados innovadores no representan una proporción significativa, siendo que sólo el $10 \%$ de la muestra (el $16 \%$ de aquellas que realizan $\mathrm{Al}$ ) expresa obtener innovaciones. No obstante, el $45 \%$ de las empresas que efectúa esfuerzos manifiesta obtener importantes alcances en términos de eficiencia y tiempos de producción, reducción de costos, sistematización de los procesos, incrementos de productividad, incorporación de capacidades de gestión, diversificación de productos y optimización en el uso de recursos.

\section{Sistema Local de Innovación y Vinculaciones entre Actores}

En primer lugar, cabe destacar que la encuesta releva información sobre las vinculaciones de las firmas sólo en el caso de las que responden afirmativamente conducir Actividades Innovativas, por lo cual el análisis se elabora sobre dicho conjunto de empresas. De aquellas firmas que realizan esfuerzos tecnológicos el $66 \%$ (37 observaciones) mantiene conexiones con otros actores, de las cuales el $70 \%$ corresponde a emprendimientos extra-locales y se encuentran distribuidas $46 \%$ en Chilecito, $38 \%$ en Arauco, y sólo el $16 \%$ en Capital.

En particular, la proporción de firmas que se vincula se eleva con el tamaño de las mismas, de las microempresas lo hace el $63 \%$, de las pequeñas el $60 \%$, de las medianas un $67 \%$, mientras que para grandes empresas dicho porcentaje alcanza al $86 \%$. A su vez, se destaca que de aquellas que obtienen resultados innovadores el $78 \%$ establece relaciones con organizaciones del SLI. En promedio las firmas establecen cuatro vinculaciones, no habiendo diferencias entre diverso origen de la inversión o departamento, pero sí en función del tamaño.

La distribución del total de articulaciones (154 vínculos) profundiza la distinción del comportamiento entre las firmas locales y las de capital nacional, siendo que éstas reúnen el $71 \%$. A su vez, el estrato con mayores interconexiones es el de firmas medianas de capitales nacionales que concentra el $30,5 \%$, seguido por las microempresas provinciales con el 26,6\% (explicado por el rol del Instituto Nacional de Tecnología Agropecuaria -INTA), y las firmas grandes extra-locales con el 20,8\% (Gráfico №2). 


\section{Gráfico N². Distribución de las Vinculaciones por Tamaño y Origen de la Inversión}

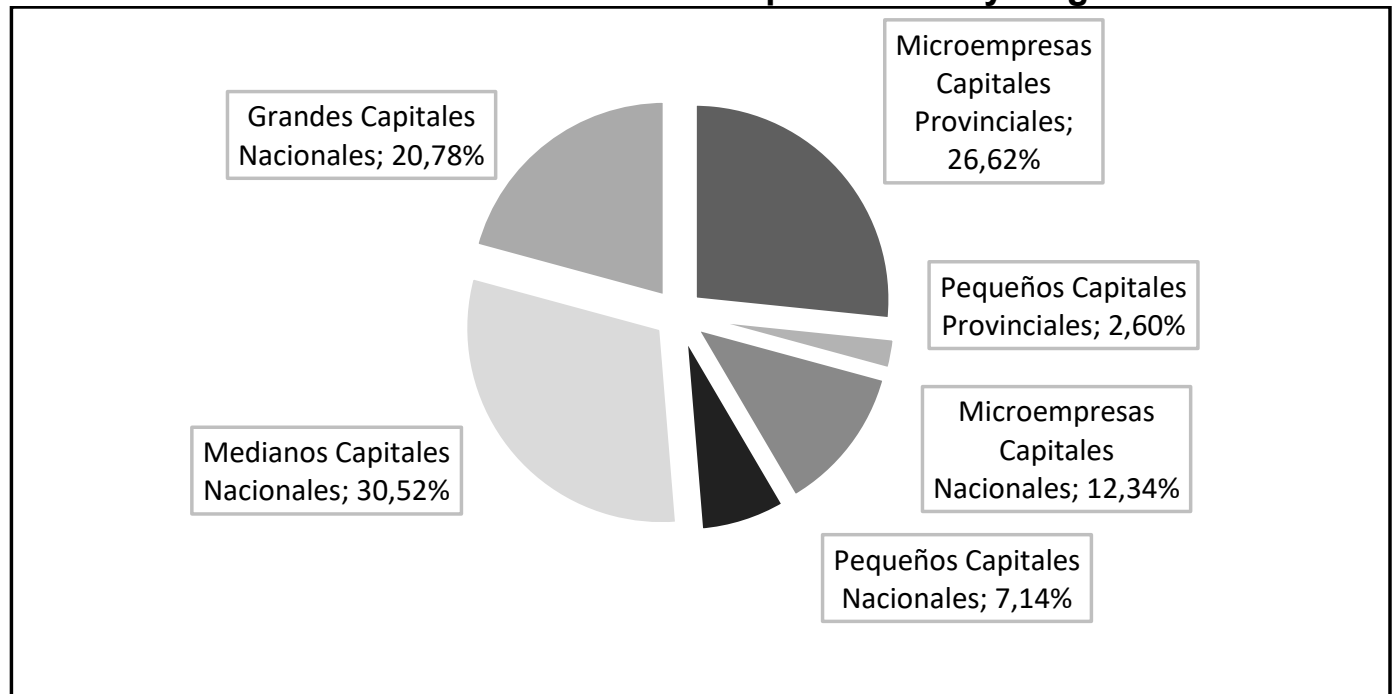

Fuente: elaboración propia en base a Encuesta "Demanda Tecnológica para el Sector Olivícola de la Provincia de La Rioja".

El actor con el que se mantiene mayor cantidad de vínculos es el INTA, el cual concentra el $30 \%$, luego, las empresas se relacionan con universidades $(15 \%)$ (Universidad Nacional de La Rioja -UNLAR-, la Universidad Nacional de Chilecito UNDEC-, Facultad Regional Universidad Tecnológica Nacional -UTN-), casa matriz (15\%), clientes y proveedores $(14 \%)$ y otras empresas $(12 \%)$. A su vez, se identifica una menor interacción con el Instituto Nacional de Tecnología Industrial-INTI- (6\%) y otros organismos de CyT (8\%) (Centro Regional de Investigaciones Científicas y Transferencia Tecnológica La Rioja - Consejo Nacional de Investigaciones Científicas y Técnicas -CRILARCONICET-, Secretaria de Ciencia y Técnica Provincial, y Consejo Federal de Ciencia y Tecnología -COFECYT-). En cuanto a la composición por tipo de vinculación, se observa que preponderan aquellas de menor complejidad, del total $39 \%$ se trata de Intercambio de Información, 17\% Ensayos y Análisis, 16\% Capacitación, 15\% Investigación y Desarrollo, y el restante $13 \%$ Asistencia Técnica.

\section{Principales Obstáculos para Realizar Actividades de Innovación y Demandas Tecnológicas}

Adicionalmente, la encuesta contiene información sobre los obstáculos que enfrentan las firmas del sector para realizar actividades de innovación. Así, entre las principales limitaciones para emprender dichos procesos se destaca, en primer lugar, el costo de adquisición de nuevas tecnologías, siendo que un $76 \%$ le asigna una importancia alta. En segundo y tercer lugar se presenta la dificultad de acceso al financiamiento (56\%) y las falencias en las políticas públicas de promoción (47\%). Entre aquellas de menor valoración 
se encuentran la rigidez organizacional, la escasa cooperación y el sistema de propiedad intelectual.

En concordancia con el análisis precedente, se observa que para todos los factores existe una mayor proporción de firmas locales que atribuyen una valoración alta. Hacia el interior de dicho grupo el $84 \%$ le otorga la máxima categoría a los costos de la tecnología, el $70 \%$ al acceso al financiamiento, el $61 \%$ al tamaño de la explotación, un $55 \%$ a los costos de capacitación y a las falencias de políticas públicas, y $52 \%$ a la falta de información sobre mercados.

Tomando en cuenta la valoración de cada obstáculo por departamento se identifican diferencias entre ellos. Las mayores divergencias se presentan en Capital, distinguiéndose la relevancia de factores relacionados al SLI como las falencias de las políticas públicas de promoción de CTI (60\%) y la escasa presencia de instituciones (60\%); así como la falta de personal calificado (53\%).

Respecto a las discrepancias entre empresas de distinto tamaño se observa que las firmas grandes manifiestan alta valoración a la dificultad de acceder a financiamiento $(80 \%)$, el costo de la tecnología (80\%), la falencia de las políticas de promoción (60\%) y la escasa cooperación con actores (40\%). Entre las microempresas se distinguen el acceso al financiamiento (63\%), la información sobre mercados (53\%), el costo de la tecnología (85\%), el dinamismo del sector (40\%), y la falencia de políticas (52\%) como los obstáculos más valorados. Tomando en cuenta la articulación con los actores del SLI, se puede mencionar que las firmas que no se vinculan señalan para todas las limitaciones mayor relevancia.

Particularmente, la dificultad de acceso al financiamiento, la falencia de las políticas públicas de promoción de CTI, y de organismos de CyT, sobresalen entre los obstáculos de mayor relevancia para el conjunto de los productores, siendo éstos características del sistema local. Sin embargo, existe un conjunto de organismos e instrumentos de promoción productiva y tecnológica, ya sean de carácter nacional o regional, disponibles para todas las empresas que son escasamente aprovechados, e incluso desconocidos por parte de la mayoría de los productores locales. De esta forma, sobresale como una debilidad de suma relevancia del SLI la falta de difusión y aprovechamiento de las políticas e instrumentos de promoción y financiamiento disponibles.

\section{c) Modelos de Respuesta Cualitativa ${ }^{1}$}

El análisis estadístico de las hipótesis planteadas se efectúa por medio de la construcción de modelos econométricos que permiten manipular variables discretas, denominados Modelos de Respuesta Cualitativa (MRC). El modelo Logit Binomial, y la

\footnotetext{
${ }^{1}$ Para un mayor desarrollo de las técnicas econométricas implementadas ver Greene (1999); Wooldridge (1997) y Cañadas Reche (2013).
} 
regresión de Quasi-Poisson, permiten estudiar la relación entre variables dependientes binarias y datos de recuento respectivamente, con un conjunto de regresores explicativos, los cuales pueden ser tanto de naturaleza cualitativa como cuantitativa. La expresión general de dichos modelos se puede plantear de la siguiente manera (Greene 1999).

$$
\operatorname{Prob}(\text { ocurra suceso } j)=\operatorname{Prob}(Y=j)
$$

Prob (ocurra suceso $j)=F$ [efectos relevantes: parámetros]

Luego, para evaluar el ajuste global de los modelos se calculan medidas que se asemejan conceptualmente al $R^{2}$ de la regresión lineal (el cual representa la proporción de la variación de $\mathrm{Y}$ que se encuentra explicada por aquella de los regresores), denominados por ello pseudo $R^{2}$. En este caso se emplearán tres medidas de bondad de ajuste de los modelos: Pseudo $\mathrm{R}^{\wedge} 2$ de McFadden, Pseudo $\mathrm{R}^{\wedge} 2 \mathrm{de}$ Cox y Snell yPseudo $\mathrm{R}^{\wedge} 2 \mathrm{de}$ Nagelkerke (Cañadas Reche, 2013).

Para el abordaje del análisis econométrico se construyen, en base a información de la muestra, diversas variables. Como variables dependientes se toman, por un lado, una variable dummy que adquiere el valor 1 si la firma conduce al menos una Actividad Innovativa, y 0 si no lo hace, la cual se analiza con un Modelo Logit Binomial. Por otro lado, se considera la Cantidad de Actividades de Innovación que efectúa la firma como variable cuantitativa discreta, la cual se incorpora en el Modelo Quassi-Poisson. En el caso de las variables independientes se tienen en cuenta las características propias de las firmas, la cantidad de vinculaciones que establecen hacia el interior del SLI y los obstáculos que perciben respecto al funcionamiento de dicho sistema (Cuadro $N^{\circ} 1$ ).

Cuadro $N^{\circ} 1$ Codificación de Variables

\begin{tabular}{lll}
\hline \multicolumn{1}{c}{ Nombre de la Variable } & \multicolumn{1}{c}{$\begin{array}{c}\text { Tipo de } \\
\text { Variable }\end{array}$} & \multicolumn{1}{c}{ Codificación } \\
\hline Departamento & Factor & $\begin{array}{l}\text { 1: Chilecito } \\
\text { 2: Arauco } \\
\mathbf{3} \text { : Capital (categoría base) }\end{array}$ \\
\hline Inversión & Dicotómica & $\begin{array}{l}\text { 1: Capitales Nacionales } \\
\text { 0: Capitales Provinciales }\end{array}$ \\
\hline Sector & Dicotómica & $\begin{array}{l}\text { 1: Sector secundario } \\
\text { 0: Sector primario }\end{array}$ \\
\hline Rango Empleados & Factor & $\begin{array}{l}\text { 1: Grandes } \\
\text { 2: Medianas } \\
\text { 3: Pequeñas } \\
\text { 4: Microempresas (categoría } \\
\text { base) }\end{array}$ \\
\hline Proporción profesionales & Cuantitativa & $\begin{array}{l}N^{\circ} \text { profesionales / Total empleados } \\
\text { Cantidad de Actividades de }\end{array}$ \\
Innovación & Cuantitativa & $\begin{array}{l}\text { Cantidad de Actividades de } \\
\text { Innovación efectuadas }\end{array}$ \\
\hline Cantidad de Vinculaciones & Cuantitativa & $\begin{array}{l}\text { Cantidad total de vinculaciones } \\
\text { establecidas }\end{array}$ \\
\hline
\end{tabular}




\begin{tabular}{lll}
\hline $\begin{array}{l}\text { Obstáculo 1 Escases de personal } \\
\text { calificado }\end{array}$ & Dicotómica & $\begin{array}{l}\text { 1: Percibe el Obstáculo } \\
\text { 0: No percibe el Obstáculo }\end{array}$ \\
\hline Obstáculo 2 Riesgo de Innovar & Dicotómica & $\begin{array}{l}\text { 1: Percibe el Obstáculo } \\
\text { 0: No percibe el Obstáculo }\end{array}$ \\
\hline $\begin{array}{l}\text { Obstáculo } 3 \text { Insuficiente } \\
\text { información sobre mercados }\end{array}$ & Dicotómica & $\begin{array}{l}\text { 1: Percibe el Obstáculo } \\
\text { 0: No percibe el Obstáculo }\end{array}$ \\
\hline $\begin{array}{l}\text { Obstáculo 4 Dificultad acceso al } \\
\text { financiamiento }\end{array}$ & Dicotómica & $\begin{array}{l}\text { 1: Percibe el Obstáculo } \\
\text { 0: No percibe el Obstáculo }\end{array}$ \\
\hline $\begin{array}{l}\text { Obstáculo 5 Escasas } \\
\text { posibilidades de cooperación }\end{array}$ & Dicotómica & $\begin{array}{l}\text { 1: Percibe el Obstáculo } \\
\text { 0: No percibe el Obstáculo }\end{array}$ \\
\hline $\begin{array}{l}\text { Obstáculo 6 Insuficiente } \\
\text { información sobre tecnología }\end{array}$ & Dicotómica & $\begin{array}{l}\text { 1: Percibe el Obstáculo } \\
\text { 0: No percibe el Obstáculo }\end{array}$ \\
\hline $\begin{array}{l}\text { Obstáculo 7 Falencias políticas } \\
\text { públicas de CyT }\end{array}$ & Dicotómica & $\begin{array}{l}\text { 1: Percibe el Obstáculo } \\
\text { 0: No percibe el Obstáculo }\end{array}$ \\
\hline $\begin{array}{l}\text { Obstáculo 8 Escaso desarrollo } \\
\text { instituciones de CyT }\end{array}$ & Dicotómica & $\begin{array}{l}\text { 1: Percibe el Obstáculo } \\
0: \text { No percibe el Obstáculo }\end{array}$ \\
\hline $\begin{array}{l}\text { Obstáculo } 9 \text { Sistema de } \\
\text { propiedad intelectual }\end{array}$ & Dicotómica & $\begin{array}{l}\text { 1: Percibe el Obstáculo } \\
0: \text { No percibe el Obstáculo }\end{array}$ \\
\hline $\begin{array}{l}\text { Obstáculo 10 Altos costos de } \\
\text { capacitación }\end{array}$ & Dicotómica & $\begin{array}{l}\text { 1: Percibe el Obstáculo } \\
0: \text { No percibe el Obstáculo }\end{array}$ \\
\hline
\end{tabular}

\section{d) Resultados Econométricos}

Los dos modelos construidos permiten establecer resultados econométricos sobre las relaciones entre las variables bajo análisis con un determinado nivel de confianza estadística, y ambos cuentan con buenas medidas de ajuste global (Tablas $\mathrm{N}^{\circ} 1$ y $\mathrm{N}^{\circ} 2$ ). Así, el Modelo $\mathrm{N}^{\circ} 1$ presenta la influencia de las variables explicativas sobre la probabilidad que tiene la firma de efectuar al menos una Actividad de Innovación. En primer lugar, el origen de la inversión resulta una variable positiva y significativa, indicando que aquellas firmas de capitales extra-locales poseen mayores oportunidades de conducir por lo menos una Al. Por su parte, los obstáculos $\mathrm{N}^{\circ} 1$ "Escasez de personal calificado" y $\mathrm{N}^{\circ} 3$ "Insuficiente información sobre mercados" presentan un efecto negativo sobre el comportamiento tecnológico, indicando que dichas debilidades del SLI afectan el accionar de las firmas del sector olivícola (Tabla $\mathrm{N}^{\circ} 1$ ).

Tabla N¹ Modelo I Logit Binomial - Conducción de Actividades de Innovación Modelo I =>AI $\sim$ dep $+i n v+$ rangoempl + prof + sector +obsta $1+o b s t a 2+o b s t a 3+$
obsta $4+o b s t a 5+o b s t a 6+o b s t a 7+o b s t a 8+o b s t a 9+o s b t a 10$

\begin{tabular}{lrrrrr}
\hline Coeficientes: & Estimación & Error Est. & Valor z & $\operatorname{Pr}(>|z|)$ & $\begin{array}{c}\text { Niv. } \\
\text { Signif. }\end{array}$ \\
\hline (Intercepto) & -1.302 & 1.669 & -0.780 & 0.435 & \\
Chilecito & 2.092 & 1.349 & 1.551 & 0.121 & \\
Arauco & 1.093 & 1.085 & 1.007 & 0.314 & \\
Inversión & 2.880 & 0.929 & 3.101 & 0.002 & $*$ \\
Grandes & 15.405 & 1406.854 & 0.011 & 0.991 & \\
Medianas & 0.228 & 0.991 & 0.230 & 0.818 & \\
Pequeñas & -1.121 & 1.072 & -1.046 & 0.296 & \\
Profesionales & 0.191 & 0.702 & 0.272 & 0.785 & \\
\hline
\end{tabular}




\begin{tabular}{|c|c|c|c|c|c|}
\hline Sector & 0.498 & 0.836 & 0.595 & 0.552 & \\
\hline Obsta1 & -1.690 & 0.930 & -1.817 & 0.069 & o \\
\hline Obsta2 & 1.332 & 0.961 & 1.385 & 0.166 & \\
\hline Obsta3 & -2.334 & 1.150 & -2.029 & 0.042 & * \\
\hline Obsta4 & -0.447 & 0.903 & -0.495 & 0.621 & \\
\hline Obsta5 & -1.358 & 1.079 & -1.259 & 0.208 & \\
\hline Obsta6 & 0.306 & 1.333 & 0.230 & 0.818 & \\
\hline Obsta7 & 1.004 & 1.211 & 0.830 & 0.407 & \\
\hline Obsta8 & 0.107 & 1.118 & 0.096 & 0.924 & \\
\hline Obsta9 & 0.819 & 0.999 & 0.819 & 0.413 & \\
\hline Obsta10 & 0.702 & 1.260 & 0.558 & 0.577 & \\
\hline
\end{tabular}

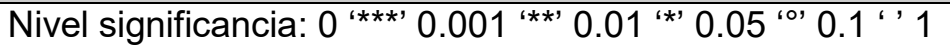

Observaciones 91

Pseudo R2: $R^{2}=0,39-R_{C N}^{2}=0,41-R_{N}^{2}=0,55$

Luego, el Modelo $\mathrm{N}^{\circ} 2$ establece la relación entre las variables independientes y la probabilidad que las firmas lleven a cabo mayor número de actividades innovativas (teniendo en cuenta la sub-muestra de aquellas empresas que realizan al menos una Al). Los resultados arrojan que la ubicación en el Departamento Chilecito, el tamaño de la empresa, la proporción de profesionales y la cantidad de vínculos que establece la firma hacia el interior del SLI son variables significativas con una influencia directa sobre el dinamismo tecnológico de las firmas (cantidad de Al). En relación a los obstáculos analizados, el $\mathrm{N}^{\circ} 2$ "Riesgo de innovar" también presenta signo positivo, lo que se asocia a que aquellas empresas más dinámicas tienen mayor percepción de su riesgo. Por el contrario, los obstáculos $\mathrm{N}^{\circ} 4$ "Dificultad de acceso al financiamiento" y $\mathrm{N}^{\circ} 6$ "Insuficiente información sobre tecnología" resultan significativos pero en este caso impactan negativamente sobre el accionar de las firmas (Tabla $\mathrm{N}^{\circ} 2$ ).

Tabla N² Modelo II Quasi-Poisson - Cantidad de Actividades de Innovación Modelo II =>cantai $\sim$ dep $+i n v+$ rangoempl + prof + sector + cantvinc + obsta $1+o b s t a 2+$ obsta $3+$ obsta $4+$ obsta $5+o b s t a 6+o b s t a 7+o b s t a 8+o b s t a 9+o s b t a 10$

\begin{tabular}{lrrrrl}
\hline Coeficientes: & Estimación & Error Est. & Valor z & $\operatorname{Pr}(>|\mathbf{z}|)$ & $\begin{array}{c}\text { Niv. } \\
\text { Signif. }\end{array}$ \\
\hline (Intercepto) & -0.261 & 0.255 & -1.023 & 0.313 & \\
Chilecito & 0.310 & 0.177 & 1.755 & 0.088 & $\circ$ \\
Arauco & 0.135 & 0.171 & 0.793 & 0.433 & \\
Inversión & 0.121 & 0.172 & 0.701 & 0.488 & \\
Grandes & 0.823 & 0.217 & 3.790 & 0.001 & $* * *$ \\
Medianas & 0.485 & 0.179 & 2.714 & 0.010 & $*$ \\
Pequeñas & 0.426 & 0.197 & 2.168 & 0.037 & $*$ \\
Profesionales & 0.572 & 0.286 & 1.997 & 0.053 & $\circ$ \\
Sector & -0.066 & 0.149 & -0.443 & 0.660 & \\
Cantidad Vínculos & 0.051 & 0.023 & 2.264 & 0.030 & $*$ \\
Obsta1 & -0.003 & 0.133 & -0.025 & 0.980 & \\
Obsta2 & 0.321 & 0.170 & 1.891 & 0.067 & $\circ$ \\
Obsta3 & 0.126 & 0.180 & 0.701 & 0.488 & \\
Obsta4 & -0.246 & 0.131 & -1.886 & 0.067 & $\circ$ \\
\hline
\end{tabular}




\begin{tabular}{|c|c|c|c|c|c|}
\hline Obsta5 & 0.311 & 0.200 & 1.555 & 0.129 & \\
\hline Obsta6 & -0.470 & 0.171 & -2.748 & 0.009 & ** \\
\hline Obsta7 & 0.078 & 0.197 & 0.395 & 0.695 & \\
\hline Obsta8 & 0.330 & 0.202 & 1.637 & 0.110 & \\
\hline Obsta9 & 0.199 & 0.181 & 1.096 & 0.281 & \\
\hline Obsta10 & -0.272 & 0.177 & -1.542 & 0.132 & \\
\hline $\begin{array}{l}\text { Nivel sigr } \\
\text { Observac } \\
\text { Pseudo F }\end{array}$ & $001^{\text {‘**’ }}$ & $0.05^{\prime}$ & & & \\
\hline
\end{tabular}

\section{Discusión y Resumen}

Entre los principales resultados se puede señalar la presencia hacia el interior del sector de firmas heterogéneas en términos productivos que presentan diversos comportamientos tecnológicos. Se trata de un sector en el que conviven productores pequeños, principalmente de capitales locales, con emprendimientos de mayor envergadura entre las que se encuentran mayormente difundidas las actividades de innovación.

En relación a la probabilidad de efectuar al menos una actividad de innovación, se destaca que son aquellas firmas de inversiones extra-locales las que poseen mayores oportunidades. Asimismo, las características del entorno local como la escasez de personal calificado y la falta de información de mercados resaltan como aspectos que tienen una influencia negativa. Respecto a la cantidad de actividades de innovación conducidas se presenta que son aquellas empresas de mayor tamaño las que cuentan con mejores probabilidades para conducirlas en mayor número, al igual que las que poseen mayor proporción de profesionales y las que se ubican en Chilecito.

Por su parte, respecto al SLI sobresale que la cantidad de vinculaciones establecidas con otros actores posee una influencia positiva sobre la posibilidad de realizar en mayor número Al. Sin embargo, la dificultad de acceso al financiamiento y la insuficiente información sobre tecnologías son factores que poseen un efecto contrario.

Así, se evidencia que hacia al interior del sector olivícola de la provincia de La Rioja el tamaño de las firmas y las debilidades del sistema local se encuentran asociados con los esfuerzos tecnológicos conducidos. En ese sentido, se observa la presencia de actores heterogéneos que no cuentan con las mismas capacidades y posibilidades para encarar actividades innovativas y de aprovechar las externalidades del entorno.

Un resultado destacado, en concordancia con los trabajos de Fritsch (2001), Fritsch y Slavtchev (2011), y Boscherini et al. (1998), es el efecto positivo que tiene la cantidad de vinculaciones sobre la probabilidad de efectuar mayor número de actividades de innovación. Se muestra así que hacia el interior del sector se presenta una relación entre las articulaciones con otros actores del SLI y los esfuerzos tecnológicos por parte de las firmas del sector. 
No obstante, los resultados que asocian el tamaño de las firmas con la cantidad de esfuerzos que realizan muestran que si bien las vinculaciones hacia el interior del SLI tienen una influencia positiva, su desarrollo no es el suficiente como para que las microempresas sorteen las barreras y limitaciones propias. Esta situación es coincidente con aquellas abordadas por Jimenez et al. (2011), ALIAS (2011) y Piñero et al. (2012) para las regiones de países latinoamericanos; Yoguel et al. (2006) en los casos de Córdoba, Rosario y las provincias del NOA; y con la investigación de Robert (2012), en las cuales se presenta una relación entre el tamaño de las firmas con sus esfuerzos, capacidades, y desempeño tecnológico.

Luego, entre las debilidades del sistema local que manifiestan las empresas del sector, se subrayan la dificultad de acceso al financiamiento y las falencias de las políticas públicas de promoción de CTI. Asimismo, sobresale el hecho de que no existen instrumentos de política locales o sectoriales específicos, y que aquellos horizontales de carácter nacional no son conocidos ni aprovechados por las firmas, lo que indica importantes fallas en la difusión de los programas vigentes. Los estudios mencionados para las regiones de Brasil, Chile y Venezuela, también revelan que las políticas de ciencia, tecnología e innovación horizontales que no reflejan las particularidades locales de cada sistema, no son eficientes para la resolución de las problemáticas específicas que afrontan (Jimenez et al., 2011; ALIAS, 2011).

Así, en función de los resultados obtenidos, y de la evidencia que presentan los antecedentes, se puede mencionar una serie de recomendaciones de política generales orientadas a promover la articulación hacia el interior del SLI y a integrar a las firmas de menor tamaño. En ese sentido, se destacan falencias en términos de diseño de políticas de CTI que limitan el alcance de las mismas a nivel regional. Éstas al no contemplar características particulares del sistema local, tales como la presencia de un débil entramado empresarial comprendido mayoritariamente por pequeños productores, la falta de recursos humanos calificados, articulaciones entre organismos de CTI y el sector productivo escasamente difundidas, menores capacidades relativas locales de generación, absorción y gestión de CTI, así como una infraestructura científica y tecnológica en desarrollo, terminan siendo escasamente aprovechadas por los actores locales.

Por otra parte, cabe destacar que el diseño de instrumentos no considera las dificultades para establecer articulaciones entre actores tales como problemas de comunicación, falta de información, tiempos disímiles e intereses contrapuestos. De igual manera, la escasa presencia de canales institucionales, vinculadores y gerentes tecnológicos que puedan facilitar el acceso a las herramientas disponibles refuerzan las limitaciones para su aprovechamiento. De este modo, aquellos instrumentos de carácter horizontal a nivel nacional no logran ser apropiados por los actores productivos y organismos de CyT que cuentan con características específicas. 
Tomando en cuenta las problemáticas identificadas se pueden proponer lineamientos para orientar el diseño de políticas en CTI que logren dar cuenta de las características territoriales. Para esto, es necesario desarrollar políticas focalizadas que apunten a la formación de capacidades de los actores locales, y a profundizar la vinculación entre los mismos.

Se deben fortalecer así las relaciones y las capacidades de los actores involucrados, en función de las particularidades que éstas revisten en la región, por lo que el proceso de planificación y diseño debe ser una instancia participativa en la cual se involucren los actores y se trabaje sobre políticas selectivas para el desarrollo local. Dentro de las mismas se pueden mencionar algunos ejemplos puntuales como la capacitación de empresarios en materia de gestión de CTI, la formación de cuadros específicos como los gerentes tecnológicos, vinculadores o los facilitadores, la capacitación de los funcionarios vinculados a la planificación de la CyT, la conformación de nuevas Unidades de Vinculación Tecnológica (UVT) en las zonas relegadas, el fortalecimiento de las UVT existentes, y el fomento permanente de instancias de trabajo conjunto entre el gobierno, representantes del sector productivo y organismos de CyT.

\section{Conclusiones Finales}

La presente investigación estudia el rol del Sistema Local de Innovación sobre el comportamiento tecnológico de las firmas para el caso del sector olivícola de la provincia de La Rioja. El propósito general es aportar a la discusión sobre las particularidades que adquiere el proceso de innovación en regiones de menor desarrollo relativo, donde los actores son heterogéneos y preponderan empresas pequeñas y medianas. Para ello se estudia la relación entre los vínculos cooperativos y las debilidades del sistema local con los esfuerzos tecnológicos conducidos por las empresas.

El análisis empírico se efectúa en base a información estadística recabada por una encuesta específica conducida a 91 productores de olivo de la provincia de La Rioja, organizada por parte de la Universidad Nacional de Chilecito en el año 2012. Luego, para el abordaje metodológico se construyen Modelos de Respuesta Cualitativa (Logit Binomial y Quassi-Poisson) para el análisis de las relaciones presentadas.

Los resultados del análisis empírico presentan evidencia a favor de las hipótesis de trabajo "Las vinculaciones de las firmas dentro del SLI las motivan a realizar actividades de innovación" y "Las debilidades presentes en el SLI representan limitaciones para las firmas respecto a su comportamiento tecnológico". Así, se observa que existe una asociación entre las interacciones, los obstáculos del SLI percibidos por las firmas y las probabilidades de conducir actividades de innovación y de llevarlas a cabo en mayor cantidad. Sin embargo, las firmas de inversiones extra-locales y de mayor tamaño cuentan con mejores probabilidades de efectuar Al y en mayor número, implicando que si bien el 
rol del SLI es relevante, no se encuentra lo suficientemente desarrollado como para que firmas de menor tamaño superen sus propias limitaciones.

Por último, se pueden proponer futuras líneas de investigación para profundizar los resultados obtenidos. Por un lado, se torna relevante indagar sobre los obstáculos que limitan las articulaciones entre las firmas del sector con organismos de CyT, y otros actores del SLI, y sobre las debilidades del sistema; para comprender de manera exhaustiva las dificultades que se presentan para establecer relaciones cooperativas, especialmente en el caso de las firmas de menor tamaño. Por otro lado, investigaciones similares pueden conducirse para diferentes regiones y localidades de Argentina, para dar cuenta de las particularidades de diversos sistemas y comparar casos de estudio, en los cuales las características de los sistemas locales y de las interacciones influyen de manera diferenciada sobre la conducta innovativa de las empresas.

Adicionalmente, pueden integrarse diversos enfoques metodológicos para enriquecer el análisis de la dinámica de los sistemas locales de innovación así como de los procesos de desarrollo tecnológico en regiones de menor desarrollo relativo. Entre ellos se destacan el análisis de redes, que da cuenta de las características de la estructura y funcionamiento del sistema, conjuntamente con estudios de cluster para analizar las ventajas y externalidades generadas en entornos de aglomeración de empresas y organismos de CTI.

En cuanto a un abordaje cuantitativo se pueden emplear diversos modelos econométricos tales como MCR, regresiones y análisis de datos multivariados, en caso de contar con series de tiempo de indicadores de innovación de entornos locales que permitan establecer relaciones entre variables con cierto nivel de confianza estadística (como por ejemplo para indagar sobre los factores que influyen en la colaboración entre firmas y otros actores del SLI). Asimismo, puede resultar de suma relevancia triangular las metodologías cuantitativas con aquellas de carácter cualitativo para dar cuenta de la dimensión subjetiva de los actores involucrados en dichos procesos, por medio de un abordaje sobre la base de entrevistas en profundidad conducidas a diversos actores locales tales como empresas, investigadores, profesionales y técnicos de organismos de CTI, y funcionarios de gobierno.

En ese sentido, se destacan un conjunto de trabajos de investigación teóricos y empíricos que profundizan la comprensión del funcionamiento, desempeño e impacto sobre el desarrollo local de los sistemas de innovación a nivel nacional, regional e internacional. En el caso de Argentina, investigaciones como Verre et. al. (2017), Niembro (2017), Marín et. al. (2015), Borello, J. (2015), Pasciaroni (2015), Bachman (2017), y Lepratte et. al. (2016) muestran las particularidades de dinámicas locales de innovación.

De manera adicional, se encuentran una serie de trabajos para América Latina y Centroamérica que presentan diversas metodologías tanto cuantitativas como cualitativas 
para el abordaje del problema de investigación (Erbes y Suárez, 2016; Aguirre Bastos y Aliaga Lordeman, 2014; Padilla Pérez, 2013; Zuniga, 2016; Rodríguez y Villarreal Peralta, 2017; Mazzucato y Penna, 2016). Por último, a nivel internacional también es posible resaltar un amplio conjunto de estudios que analizan en profundidad las características de los procesos mencionados a nivel regional como Klein y Sauer (2016); Porto Gómez et. al. (2016), Trippl et. al. (2015), Alberdi Pons et. al. (2014), Lau y Lo (2015), y Ligenzowska, J. (2016). Éstos representan importantes antecedentes para futuras investigaciones y proveen tanto desarrollos teóricos como metodológicos de suma utilidad.

\section{Referencias Bibliográficas}

Aguirre Bastos, C. y J. Aliaga Lordeman (2014). The Innovation System in Bolivia: Overall Perspectives. Instituto de Investigaciones Socio-económicas. Documento de Trabajo $\mathrm{N}^{\circ} 09 / 14$.

Alberdi Pons, X., J. J. Gibaja, y M. D. Parrilli (2014). Evaluación de la fragmentación en los Sistemas Regionales de Innovación: Una tipología para el caso de España. Investigaciones Regionales; 28, 7-35.

ALIAS (2011). Diseño y Establecimiento de la Estrategia Regional de Innovación y Acciones Afines en Biobío. Proyecto Red Región de Biobío. Fondo de Innovación Tecnológica Región del Bio-Bio.

Asheim, B. T. y L. Coenen (2005). Knowledge bases and regional innovation systems: Comparing Nordic clusters. Research Policy; (34), 1173-1190.

Bachman, F. (2017). La innovación industrial y sus determinantes desde un enfoque sistémico. Faces; 23(48).

Borello, J. (2015). Geografía de la innovación en la Argentina: Primer análisis regional basado en datos sobre PYMES. En G. Peretti, N. Gómez y N. Finelli (Eds.).Tendencias y desafíos de la geografía en el siglo XXI. Santa Fe: Universidad Nacional del Litoral.

Boscherini, F., M. López, y G. Yoguel. (1998). Sistemas locales de innovación y el desarrollo de la capacidad innovativa de las firmas: un instrumento de captación aplicado al caso de Rafaela. Globalización e Innovación Localizada: Experiencias de Sistemas Locales en el Ámbito del Mercosur y Propuestas de Políticas de C\&T. Documento Nº17/98, Instituto de Economía de la Universidad Federal de Río de Janeiro.

Camagni, R. y R. Capello (1997). Innovation and Performance of SMEs in Italy: The relevance of Spatial Aspects. Centre for Business Research, University of Cambridge.

Cañadas Reche, J. L. (2013). Regresión logística. Tratamiento computacional con R. Tesis de Maestría. Granada: Facultad de Ciencias, Universidad de Granada.

Cassiolato, J. E., H. M. Lastres y M. L. Maciel (2003). Systems of innovation and development. Londres: Edward Elgar Publishing. 
David, P. y D. Foray (2002). Una introducción a la economía y a la sociedad del saber. Revista Internacional de las Ciencias Sociales; 171.

Day, J. (2013). Actualidades y desafíos en la cadena olivícola de Argentina. Documento de Trabajo, Edición №128. Fundación Mediterránea.

DNPR (2016). Fichas Provinciales 2016, La Rioja. Secretaría de Política Económica y Planificación del Desarrollo. Dirección Nacional de Planificación Regional.

Erbes A. y D. Suárez (Comps.) (2016). Repensando el desarrollo latinoamericano. Una discusión desde los sistemas de innovación. Buenos Aires: Ediciones UNGS. Universidad Nacional General Sarmiento.

Fritsch, M. (2001). Cooperation in Regional Innovation Systems. Regional Studies; 35(4), 297-307.

Fritsch, M. y V. Slavtchev (2011). Determinants of the efficiency of regional innovation systems. Regional Studies 45(7), 905-918.

Fritsch, M. y G. Franke (2004). Innovation, regional knowledge spillovers and R\&D cooperation. Research Policy; 33, 245-255.

Gennero de Rearte, A., E. Lanari, y P. Alegre (2006); Capacidad innovativa de núcleos impulsores de firmas en entornos territoriales dinámicos. El caso de Mar del Plata, Argentina. En J. Cassiolato y H. Lastres (Eds.). Experiencias de sistemas locales en el Mercosur. Brasilia: IBICT.

Greene, W. (1999). Análisis Econométrico. Tercera Edición. Madrid: Prentice Hall Iberia. Intarakumnerd, P. y J. Vang (2006). Clusters and Innovation Systems in Asia. Science Technology \& Society;11(1), 1-7.

Grillitsch, M. y M. Nilsson (2015). Innovation in peripheral regions: Do collaborationscompensate for a lack of local knowledge spillovers?. The Annals of Regional Science; 54(1), 299-321.

Jiménez F., I. Fernández de Lucio, y A. Menéndez (2011). Los Sistemas Regionales de Innovación: experiencias concretas en América. En J. Llisterri y C. Pietrobelli (Eds.). Los Sistemas Regionales de Innovación en América Latina. Buenos Aires: Banco Interamericano de Desarrollo.

Kaufman, A. y F. Tödtling (2000). Science-Industry Interaction in the Process of Innovation: The Importance of Boundary-Crossing between Systems. 40th Congress of the European Regional Science Association. Barcelona.

Klein, M. y A. Sauer (2016). Celebrating 30 years of innovation system research: What you need to know about innovation systems. Papers in Business, Economics and Social Sciences; (17). 
Lau, A. K.W. y W. Lo (2015). Regional innovation system, absorptive capacity and innovation performance: An empirical study. Technological Forecasting and Social Change. Elsevier; 92(C), 99-114.

Lepratte, L., R. Blanc y L. Miotti (2016). Innovación y desarrollo regional. Aportes a una agenda de estudios de innovación a escala de regiones en Argentina. Curitiva: XI Jornadas Latinoamericanas de Estudios Sociales de Ciencia y Tecnología.

Ligenzowska, J. (2016). Regional Innovation Systems in Sweden. Ekonomia Międzynarodowa; (16).

López, A. (2002). Industrialización Sustitutiva de Importaciones y Sistema Nacional de Innovación: Un análisis del caso Argentino. Universidad Nacional de Quilmes. Revista REDES; 10(19), 43-85.

Lundvall, B.-Å. (1997). National Systems and National Styles of Innovation. 4th International ASEAT Conference: Differences in styles of technological innovation. Manchester.

Marín, A., N. Liseras, C. Calá, y F. Graña (2015). Oportunidades de innovación divergentes: ¿es el territorio importante?. Bahía Blanca: XX Reunión Anual de Red Pymes Mercosur.

Matías A., S. Molina, V. Aybar, J. Ladux y J. Ortiz. (2012). Olivicultura Argentina y Regional. Documento de Trabajo. INTA.

Mazzucato, M. y C. Penna (2016). The Brazilian Innovation System: A Mission-Oriented Policy Proposal. Avaliação de Programas em CT\&I. Apoio ao Programa Nacional de Ciência (Plataformas de conhecimento). Brasília, DF: Centro de Gestão e Estudos Estratégicos.

Natário, M., A. Braga, J. Couto y T. Tiago (2012). Estándares Territoriales de Innovación: Análisis de las Regiones de Portugal. Revista de Estudios Regionales; (95).

Niembro, A. (2017). Hacia una primera tipología de los sistemas regionales de innovación en Argentina. Journal of Regional Research; (38), 117-149.

Padilla Pérez, R. (Ed.) (2013). Sistemas de innovación en Centroamérica. Fortalecimiento a través de la integración regional. Santiago de Chile: Comisión Económica para América Latina y el Caribe (CEPAL).

Pasciaroni, C. (2015). Organizaciones de conocimiento y sistemas regionales de innovación en países en desarrollo. Estudio de caso para argentina. Regional and Sectoral Economic Studies; 15(2).

Piñero, A., M. Arzola, C. Rodríguez Monroy (2012). Articulación del Sistema Regional de Innovación para Desarrollar Capacidad de I+D+i. I Jornadas de Conocimiento e Investigación del Área de Tecnología de Procesos Productivos, Venezuela. 
Porto Gómez, I., J. R. Otegi Olaso y J. M. Zabala-Iturriagagoitia (2016). ROSA, ROSAE, ROSIS: modelling a regional open sectoral innovation system. Entrepreneurship \& Regional Development; 28(1-2), 26-50.

Robert, V. (2012). Interacciones, feedbacks y externalidades: la micro complejidad de los sistemas productivos y de innovación locales. Una aproximación en PyMes argentinas. Tesis Doctoral. Facultad de Ciencias Económicas, Universidad de Buenos Aires.

Rodríguez, A. y E. M. Villarreal Peralta (2017). Innovation and Regional Growth in Mexico: 2000-2010. Papers in Evolutionary Economic Geography: (\#14.17).

Sánches, P. (2013). Análisis de Diagnóstico Tecnológico Sectorial - Olivarero. Estudios de Análisis de Diagnóstico Tecnológico Sectorial. Secretaría de Planeamiento y Políticas. Ministerio de Ciencia, Tecnología e Innovación Productiva.

Tödtling, F., P. Lehner, y A. Kaufmann (2008). Do different types of innovation rely on specific kinds of knowledge interactions?SRE - Discussion Papers;(01), Institut für Regional- und Umweltwirtschaft, WU Vienna University of Economics and Business.

Trippl, M., B.T. Asheim y J. Miorner (2015). Identification of regions with less developed research and innovation systems. Papers in Innovation Studies; 2015/1. Lund University, CIRCLE - Center for Innovation, Research and Competences in the Learning Economy.

UNdeC (2013). Encuesta Demanda Tecnológica para el Sector Olivícola de la Provincia de La Rioja. Unidad de Vinculación Tecnológica. Universidad Nacional de Chilecito.

Verre, V., D. Milesi y N. Petelski (2017). Estrategias de apropiación en contextos de colaboración público-privada en la biotecnología argentina. Nueva Época; (47), 31-64.

Vita Serman, F. y C. Matías (2013). Cadena Olivo. Programa Nacional Frutales. INTA.

Wooldridge, J. M. (1997). Quasi-likelihood methods for count data. Handbook of applied econometrics; (2), 352-406.

Yoguel, G., J. A. Borello, y A. Erbes. (2009). Argentina: cómo estudiar y actuar sobre los sistemas locales de innovación. Revista CEPAL; 99.

Yoguel, G., J. Borello, y A. Erbes. (2006). Sistemas Locales de Innovación. Los casos de Córdoba, Rafaela, Rosario y Tucumán, Salta y Jujuy. Programa Sistema Nacional y Sistemas Locales de Innovación. Anexo 5 Modulo B. Observatorio Nacional de Ciencia, Tecnología e Innovación.

Yoguel, G. y F. Boscherini (2001). El desarrollo de las capacidades innovativas de las firmas y el rol del sistema territorial. Desarrollo Económico; 41(161).

Zaraychenko, I. A., A. I. Shinkevich, M. Y. y Shvetsov et. al. (2016). Innovation Networks Modeling Within the Concept of Open Innovations. International Journal of Economics and Financial Issues; 6(1), 192-198.

Zuniga, P. (2016). Innovation system in development: The case of Peru. Maastricht Economic UNU-MERIT Working Papers: (\#2016-058). 